\title{
Brain Changes in Kallmann Syndrome
}

\author{
R. Manara, A. Salvalaggio, A. Favaro, V. Palumbo, V. Citton, A. Elefante, A. Brunetti, F. Di Salle, G. Bonanni,
} and A.A. Sinisi, for the Kallmann Syndrome Neuroradiological Study Group

\begin{abstract}
BACKGROUND AND PURPOSE: Kallmann syndrome is a rare inherited disorder due to defective intrauterine migration of olfactory axons and gonadotropin-releasing hormone neurons, leading to rhinencephalon hypoplasia and hypogonadotropic hypogonadism. Concomitant brain developmental abnormalities have been described. Our aim was to investigate Kallmann syndrome-related brain changes with conventional and novel quantitative MR imaging analyses.
\end{abstract}

MATERIALS AND METHODS: Forty-five male patients with Kallmann syndrome (mean age, 30.7 years; range, 9-55 years) and 23 agematched male controls underwent brain MR imaging. The MR imaging study protocol included 3D-T1, FLAIR, and diffusion tensor imaging (32 noncollinear gradient-encoding directions; b-value $=800 \mathrm{~s} / \mathrm{mm}^{2}$ ). Voxel-based morphometry, sulcation, curvature, and cortical thickness analyses and tract-based spatial statistics were performed by using Statistical Parametric Mapping 8, FreeSurfer, and the fMRI of the Brain Software Library.

RESULTS: Corpus callosum partial agenesis, multiple sclerosis-like white matter abnormalities, and acoustic schwannoma were found in 1 patient each. The total amount of gray and white matter volume and tract-based spatial statistics measures (fractional anisotropy and mean, radial, and axial diffusivity) did not differ between patients with Kallmann syndrome and controls. By specific analyses, patients with Kallmann syndrome presented with symmetric clusters of gray matter volume increase and decrease and white matter volume decrease close to the olfactory sulci; reduced sulcal depth of the olfactory sulci and deeper medial orbitalfrontal sulci; lesser curvature of the olfactory sulcus and sharper curvature close to the medial orbital-frontal sulcus; and increased cortical thickness within the olfactory sulcus.

CONCLUSIONS: This large MR imaging study on male patients with Kallmann syndrome featured significant morphologic and structural brain changes, likely driven by olfactory bulb hypo-/aplasia, selectively involving the basal forebrain cortex.

ABBREVIATIONS: $\mathrm{KS}=$ Kallmann syndrome; $\mathrm{FA}=$ fractional anisotropy; $\mathrm{MNI}=$ Montreal Neurological Institute; TBSS $=$ tract-based spatial statistics; VBM = voxel-based morphometry

K

allmann syndrome (KS) is a rare inherited disorder (affecting about 1 in 10,000 males), ${ }^{1}$ clinically characterized by the association of hypogonadotropic hypogonadism and hypo-/anosmia. ${ }^{2}$ Both KS clinical hallmarks derive from a disturbed intrauterine migration process involving olfactory axons and gonadotropin-releasing hormone neurons from the olfactory pla-

Received December 20, 2013; accepted after revision February 3, 2014

From the Department of Neuroradiology (R.M., F.D.S.), University of Salerno, Salerno, Italy; Istituto di Ricovero e Cura a Carattere Scientifico S. Camillo (R.M., V.C.) Venezia, Italy; Departments of Neurology (A.S.), and Psychiatry (A.F.), Department of Neurosciences; Unità di Endocrinologia (G.B.), Department of Medicine, University of Padova, Padova, Italy; Department of Clinical and Experimental Medicine and Surgery (V.P., A.A.S.), Endocrinology and Medical Andrology Section, Second University of Napoli, Napoli, Italy; and Department of Neuroradiology (A.E., A.B.), Department of Scienze Biomediche Avanzate, and ENT section (R.M., A.S.), Department of Neurosciences, "Federico II" University, Napoli, Italy.

Renzo Manara and Alessandro Salvalaggio equally contributed to the study and should be considered first authors. code to the hypothalamus. ${ }^{3,4}$ The failure of the migration process results in hypo-/aplasia of the rhinencephalon (olfactory bulbs and tracts $)^{3-6}$ and in altered gonadotropic axis function with low levels of sex hormones. Besides rhinencephalon abnormalities, distinctive KS neuroradiologic changes have been detected in the brain and bone structures of the anterior cranial fossa by conventional MR imaging ${ }^{7-14}$ and CT studies. ${ }^{15}$ The most known morphologic brain feature is the reduction in depth and length of the

\footnotetext{
Guglielmo Bonanni and Antoio Agostino Sinisis should be considered senior coauthors.

Paper previously presented in part at: Annual Meeting of the European Society of Neuroradiology, September 28 to October 1, 2013; Frankfurt, Germany.

Please address correspondence to Renzo Manara, MD, Neuroradiology, University of Salerno, Via S Allende 1, Baronissi 89081 (SA), Italy; e-mail: rmanara@unisa.it

三 Indicates article with supplemental on-line appendix and table.

http://dx.doi.org/10.3174/ajnr.A3946
} 
olfactory sulcus, which typically turns medially, opening anteriorly into the interhemispheric fissure. ${ }^{7-13}$ This sulcal abnormality is thought to be driven by the absence/hypoplasia of the olfactory bulbs and represents an intriguing model of genetically driven developmental brain abnormalities. Furthermore, a few case reports postulated the strict relationship between KS and midline brain abnormalities, such as corpus callosum agenesis and holoprosencephaly, ${ }^{6,16}$ though pathologic and neuroimaging data are relatively scarce and often contradictory. ${ }^{7}$

In 2008, a pioneering study by voxel-based morphometry (VBM) MR imaging analysis revealed distinct regional gray and white matter volume changes in male patients with KS outside the frontal orbital regions. ${ }^{17}$ No study so far has replicated a larger sample of these findings, which would imply a much more profound effect of KS-related genes (KAL1, FGFR1, PROK2, PROKR2, FGF8, NELF, etc. $)^{18}$ and/or sex hormone deficiency on brain morphogenesis and development. Moreover, no study has investigated regional white matter changes revealed by VBM by diffusion tensor imaging, a powerful quantitative technique able to investigate the structural nature of white matter involvement. Finally, novel MR imaging-based analyses have been developed that allow assessing precise curvature, sulcation, and cortical thickness quantitative evaluations, ${ }^{19-21}$ thus providing further insights into cortex developmental abnormalities. Such analyses have not been applied to KS, though they might represent useful tools for investigating the structural underpinnings of neurologic and psychiatric disorders anecdotally reported in patients with $\mathrm{KS}^{22,23}$

By conventional MR imaging and novel quantitative sulcation, curvature, cortical thickness, and tract-based spatial statistics (TBSS) analyses, we aimed to feature, more precisely and in a large sample of male patients, the morphologic and structural brain involvement in KS.

\section{MATERIALS AND METHODS Subjects}

Forty-five male patients (mean age, 30.7 years; range, 9-55 years) affected with KS underwent brain MR imaging. All patients met the diagnostic criteria for KS based on clinical observations and smell analysis (main clinical and demographic features are shown in On-line Table 1). One patient had a history of severe head trauma. Twenty-three healthy male subjects (mean age, 32.6 years; range: $12-55$ years) or patients referred to neuroimaging for headache with no history of prematurity, head trauma, neurologic or psychiatric disease, or neurosurgery represented our control group.

Seven patients with KS and 2 controls were left-handed, according to the Edinburgh Handedness Inventory. ${ }^{24}$ No patients or subjects required sedation during MR imaging acquisition. The study was approved by the local ethics committee, and written informed consent was obtained from patients or their parents.

Patients with KS younger than 12 years of age or presenting with midline brain abnormalities or significant parenchymal lesions were excluded from VBM, sulcation, curvature, and TBSS analyses to avoid the interference of maturation, malformative, or incidental factors on the quantitative parenchymal and morphologic evaluation.

\section{MR Imaging Acquisition}

All MR imaging scans were obtained in 2 centers (University Hospital of Padova and Medicanova Diagnostic Center, Battipaglia, Italy) equipped with the same 1.5T MR imaging scanner (Achieva; Philips Healthcare, Best, the Netherlands) with a standard quadrature head coil. The MR imaging study protocol included the following: 3D T1-weighted imaging (TR/TE, 20/3.8 ms; flip angle, 20 ; section thickness, $1 \mathrm{~mm}$; acquired pixel size, $1 \times 1 \mathrm{~mm}$; reconstructed pixel size, $0.66 \times 0.66 \mathrm{~mm}$; acquisition matrix, $212 \times 210$; reconstructed matrix, $320 \times 320$; acquisition time, approximately 7 minutes); and FLAIR (TR/TE/TI, 10,000/140/2800 ms; echo-train length, 53; flip angle, $90^{\circ}$; section thickness, $5 \mathrm{~mm}$; intersection gap, $0.5 \mathrm{~mm}$; acquisition pixel, $0.90 \times 1.15 \mathrm{~mm}$; reconstructed pixel, $0.9 \times 0.9 \mathrm{~mm}$; acquisition time, 3 minutes 20 seconds).

Diffusion tensor images were acquired with single-shot echoplanar diffusion-weighted imaging (TR/TE, 11,114/80 ms; acquisition matrix, $112 \times 110$; echo-train length, 59; reconstructed matrix, $128 \times 128$; acquisition pixel, $2 \times 2 \mathrm{~mm}$; reconstructed pixel, $1.75 \times 1.75 \times 2 \mathrm{~mm}$; sensitivity encoding $\mathrm{p}$ reduction, 2 ; section thickness, $2 \mathrm{~mm}$ without gap; NEX, 2; acquisition time, 12 minutes 24 seconds). The axial sections covered the whole brain including the cerebellum. The diffusion-sensitizing gradients were applied along 32 noncollinear gradient-encoding directions with maximum $b=800 \mathrm{~s} / \mathrm{mm}^{2}$. One additional image without diffusion gradients $\left(b=0 \mathrm{~s} / \mathrm{mm}^{2}\right)$ was also acquired.

\section{Image Processing}

Data Processing of Volumetric Images and DTI (TBSS). The imaging processing methods are presented in detail in the On-line Appendix.

Specifically, we used the optimized VBM protocol (Diffeomorphic Anatomical Registration Through Exponentiated Lie Algebra) available in the statistical parametric mapping software (SPM8; Wellcome Department of Imaging Neuroscience, London, UK, www.fil.ion.ucl.ac.uk/spm) ${ }^{25}$ For statistical analyses, we used parametric $t$ tests as implemented by SPM8, by using age as a covariate of no interest. Results for gray matter were considered significant for $P<.05$, family-wise error-corrected.

All 3D T1-weighted data were also processed by FreeSurfer (http://surfer.nmr.mgh.harvard.edu/) to derive quantitative estimates of cortical thickness, sulcation, and curvature. The sulcation conveys information on how far a particular surface vertex point is from a hypothetic "midsurface," which exists between the gyri and sulci. The curvature conveys information on the curvature (not distance) at a specific vertex point. The sharper the curve, the higher the value (positive or negative) is. The color conveys the sign and is just an arbitrary choice. Cortical thickness, sulcation, and curvature were estimated for the whole brain. Group analysis was performed by using generalized linear models, including age as a nuisance variable. Results were considered significant for $P<.05$, false discovery rate-corrected.

All DTI data were preprocessed by the FMRIB Diffusion Toolbox within FSL (http://www.fmrib.ox.ac.uk/fsl/fdt/index.html). ${ }^{26}$ For group comparisons concerning fractional anisotropy (FA) and diffusivity values, data were fed into the voxelwise statistics analysis, which was based on nonparametric permutation testing (5000 permutations) by using the threshold-free cluster enhance- 

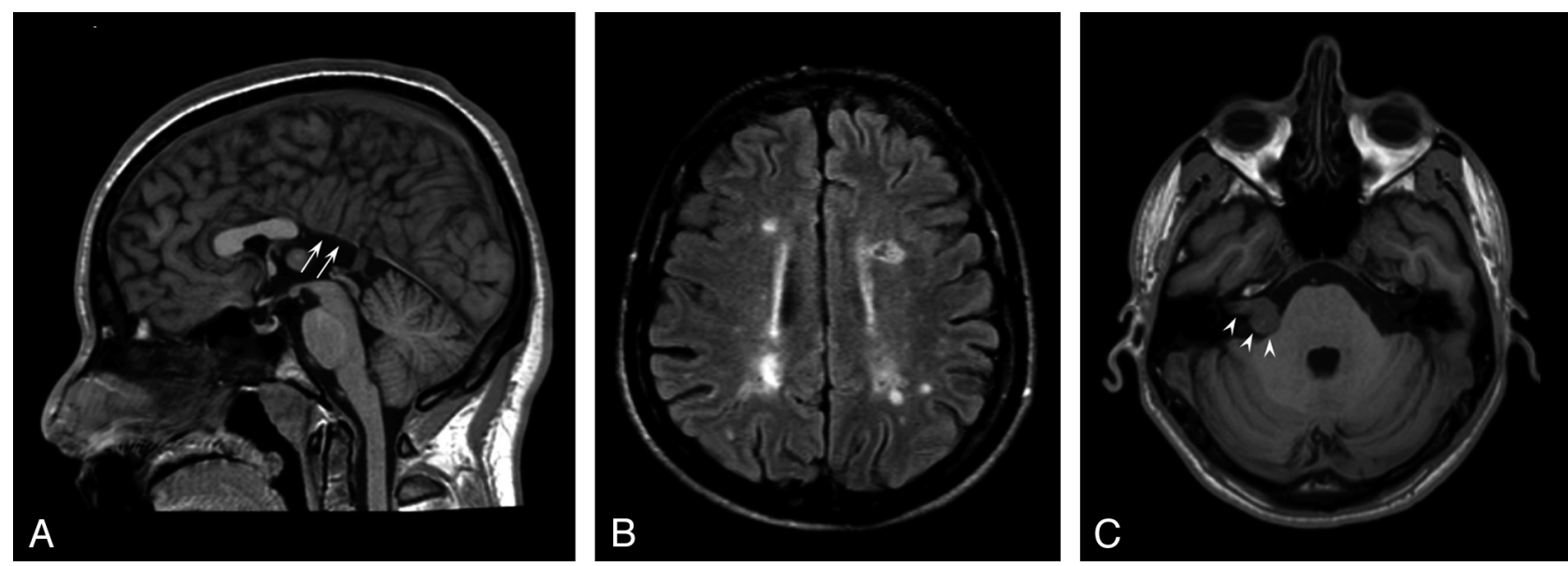

FIG 1. Conventional brain MR imaging findings in 45 patients with Kallmann syndrome. A, Midsagittal T7-weighted image of a patient with agenesia of the posterior portion of the corpus callosum (white arrows). B, Axial FLAIR image of the patient disclosing several multiple sclerosis-like white matter signal abnormalities in the centrum semiovale bilaterally. C, Non-enhanced axial T1-weighted image at the level of the internal acoustic meatus showing a right intra/extrameatal dumbell-shaped mass consistent with an acoustic schwannoma.

ment ${ }^{27}$ method to account for multiple comparison correction across space. Age and sites of MR imaging acquisition were entered into the analysis as covariates.

\section{RESULTS}

\section{Conventional MR Imaging Findings Evaluation}

One patient with KS had midline brain abnormalities (partial corpus callosum agenesia) (Fig 1A), and 1 had diffuse multiple sclerosis-like white matter abnormalities (Fig $1 B$ ).

On FLAIR images, nonspecific white matter signal abnormalities (1-20 punctuate hyperintensities) were observed in 15/45 patients (33\%; mean age, 35.3 years; age range, $15-55$ years); age did not differ between patients with and without white matter hyperintensities $(P=.07)$. Similarly, the presence of obesity ( $7 /$ $45)$, hypertension (0/45), and cardiopathy (0/45) did not differ between these 2 subgroups.

One patient had small cortical abnormalities in the right superior frontal and inferior temporal gyri, consistent with sequelae of a known previous head trauma. One patient had a right intra/ extrameatal acoustic nerve schwannoma $(20 \times 14 \mathrm{~mm})$, with no significant mass effect on the pons (Fig 1C).

\section{Quantitative MR Imaging Analysis}

The 3 patients with partial agenesis of the corpus callosum, diffuse multiple sclerosis-like white matter lesions, and age younger than 12 years were excluded from quantitative analyses. Forty-two patients with $\mathrm{KS}$ were, therefore, considered for subsequent analyses (mean age, 30.6 years; range, $15-55$ years).

\section{Voxel-Based Morphometry}

The total amount of gray and white matter did not differ between patients with KS and controls (gray matter, $919 \pm 132 \mathrm{~mL}$ versus $949 \pm 108 \mathrm{~mL}$; white matter, $677 \pm 091 \mathrm{~mL}$ versus $710 \pm 081 \mathrm{~mL}$, respectively) by using as covariates age, total intracranial volume, and site of MR imaging.

Patients with KS showed, compared with controls, 2 almost symmetric clusters of significant white matter volume decrease in the frontal basal regions (Montreal Neurological Institute
[MNI] coordinates: $-16,26,-26$ and $14,32,-28 ; P$ value family-wise error-corrected, respectively, $<.001$ and .002), 2 almost symmetric clusters of significant gray matter volume increase in the frontal basal regions (MNI coordinates: 16, 38, -12 and $-16,34,-12$; $P$ value family-wise error-corrected, .001 ), and 2 almost symmetric clusters of significant gray matter volume decrease in the frontal basal regions (MNI coordinates: $-10,32,-24$ and $12,26,-18$; $P$ value family-wise error-corrected <.001) by using as covariates age, total intracranial volume, and site of MR imaging. As shown in Fig 2 , these regions were very close to the olfactory sulci. Patients with KS did not show clusters of significant white matter volume increase compared with controls.

\section{Sulcation, Curvature, and Cortical Thickness Whole-Brain Analyses}

Significant differences between male patients with KS and controls are shown in the Table and Fig 2, in particular in the following manner:

By sulcation analysis, the sulcal depth was reduced at the level of the olfactory sulci and increased at the level of the medial orbital-frontal sulci in patients with KS.

By curvature analysis, patients with KS presented with a decreased curvature in the olfactory sulcus and a sharper curvature close to the medial orbital-frontal sulcus, bilaterally.

By cortical thickness analysis, patients with KS had thicker cortices than controls in a region corresponding to the olfactory sulcus, bilaterally. In the left hemisphere, 2 small areas of decreased cortical thickness were found at the level of the medial and lateral orbital-frontal sulci, the larger close to the olfactory sulcus.

\section{Tract-Based Spatial Statistics Analysis}

TBSS whole-brain analysis showed no differences between patients with KS and controls regarding fractional anisotropy, mean diffusivity, radial diffusivity, and axial diffusivity. 

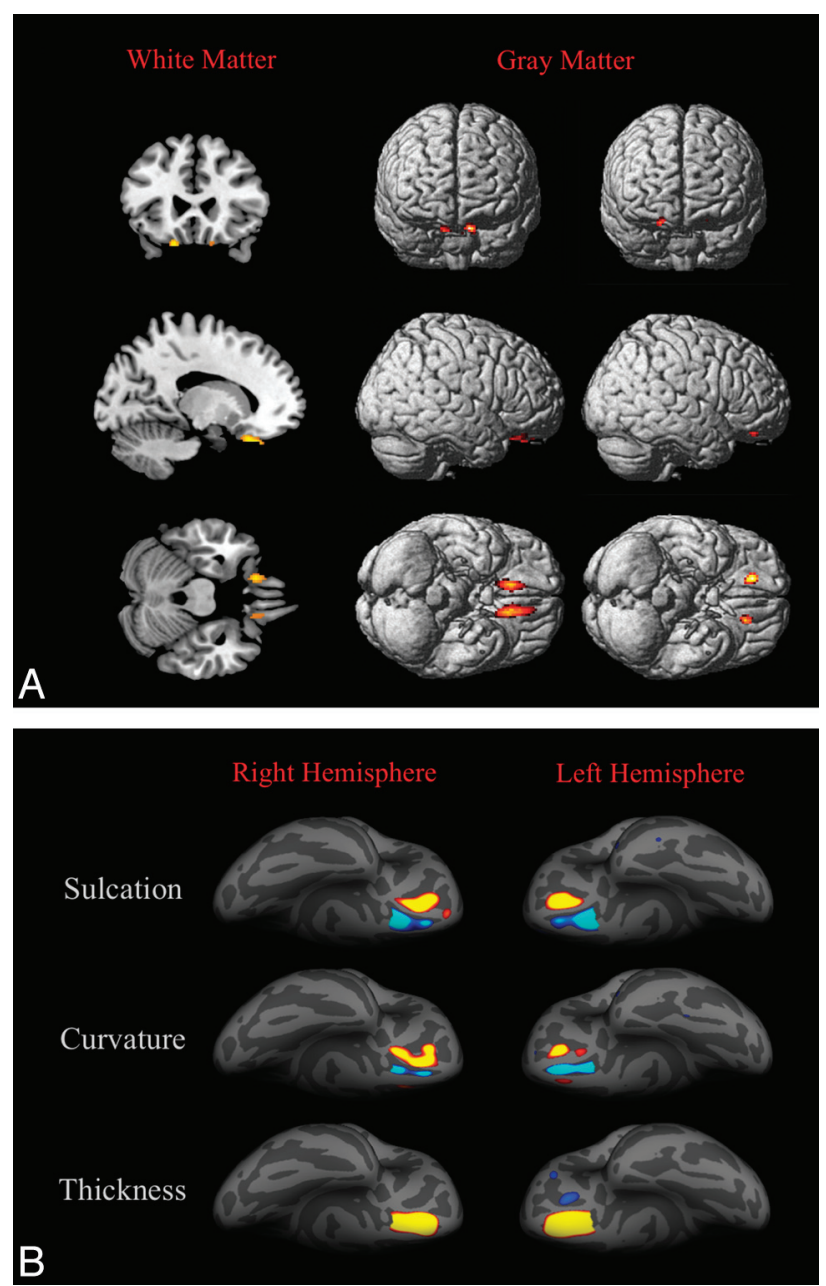

FIG 2. 3D-T1-based whole-brain analyses on 42 patients with Kallmann syndrome versus 23 controls. A, Voxel-based morphometry findings. Clusters of significantly decreased white matter volume (colored areas in the multiplanar reconstructions in the first column) were detected exclusively and symmetrically in the posterior portion of the medial orbital-frontal gyrus close to the olfactory sulcus; no regions of increased white matter volume were detected in our sample. Clusters of significantly decreased (second column) and increased (third column) gray matter volume are shown as colored cortical areas in the volume-rendering technique images within or close to the olfactory sulci. $B$, Sulcation, curvature, and thickness findings. Colored areas represent increased (yellow-red) and decreased (blue) values in patients with KS. Almost all differences are clustered within the olfactory sulci and the neighboring cortex of the rectus and medial orbital-frontal gyri.

\section{DISCUSSION}

The present study showed that patients with KS display surface cortical variations and gray and white matter volume changes, which clustered symmetrically in the frontal basal regions, close to the olfactory bulbs (gyrus rectus, medial orbital-frontal gyrus).

Changes in the frontal basal regions have been consistently described in the rhinencephalon and contiguous cerebral cortical and bone structures by pathology, ${ }^{5,6}$ conventional MR imaging, ${ }^{7-14}$ and CT studies in patients with KS. ${ }^{15}$ Increased volume of the corticospinal tracts and corpus callosum has been also depicted by conventional MR imaging, ${ }^{28}$ suggesting the involvement of brain structures beyond the basal forebrain, though these findings have not been confirmed in larger series. ${ }^{7,17}$ A single MR imaging study formerly applied VBM to investigate gray and white matter volume in male patients with KS, detecting changes in several areas outside the basal forebrain, namely regions of increased gray matter volume (precentral gyrus bilaterally, left middle occipital and middle frontal gyri), decreased gray matter volume (right parahippocampal gyrus), increased white matter volume (left cuneus and right subcallosal gyrus), and decreased white matter volume (left superior frontal gyrus, right medial frontal gyrus, left frontal lobe subgyral and left insula). ${ }^{17}$ In our VBM whole-brain analysis, gray and white matter changes appeared fairly symmetric and limited to the frontal basal regions. White matter volume changes (ie, decrease of white matter volume) were found exclusively in small subcortical areas of the medial orbital-frontal gyri close to the olfactory sulci. Similarly, VBM analysis disclosed small symmetric and contiguous areas of increased and decreased gray matter volume in the frontal basal regions close to the olfactory sulcus. The striking relationship between white and gray matter changes seems to suggest that these changes embody a fairly localized cortical-subcortical abnormal architectural development. Moreover, because all parenchymal changes are very close to the olfactory sulcus, they are most likely induced by the rhinencephalon hypo-/aplasia.

Except for these areas, by VBM analysis, cerebral gray and white matter did not differ between patients with KS and healthy controls. Similarly, TBSS analysis did not reveal any significant difference between patients with KS and controls, revealing that the ultrastructure of the white matter is preserved despite significant forebrain cortical changes. Nonetheless, KS genetic heterogeneity does not allow the exclusion of white matter hypertrophic or degenerative phenomena in specific subgroups of patients with KS because a strict genotypic/MR imaging phenotypic correlation requires a larger sample. Actually, no pathologic study reported brain parenchyma abnormalities in patients with KS, even though histologic data are very scarce. Peripheral axonal degeneration due to the absence of the olfactory bulbs has been shown in the olfactory mucosa, ${ }^{29}$ but concomitant significant processes of axonal degeneration within the brain are not supported by VBM and TBSS findings.

Differences from previous MR imaging studies may be due to several issues, among them, the heterogeneity of the phenotypic spectrum of brain anomalies in KS or a different methodologic approach. The size of our study sample and the spatial coherence between gray/white matter volume changes and the well-known disease-related abnormalities of the olfactory sulcus seem to support the findings of the present study.

Consistently, the whole-brain curvature and sulcation analyses showed symmetric cortical abnormalities strictly confined to the frontal basal cortical regions (gyri recti and medial orbitalfrontal gyri). Olfactory sulcus abnormalities were expected because the reduction in depth and length of the olfactory sulcus at gross pathology and conventional MR imaging evaluation is a known morphologic feature of the brain in patients with KS. ${ }^{7-12}$ Olfactory sulcus abnormalities have been associated with embryogenic olfactory bulb-inducted processes. ${ }^{10,11,30,31}$ In patients with KS, the aplasia/hypoplasia of the olfactory bulbs is associated with an ipsilaterally decreased depth of the olfactory sulcus, which might turn medially, opening anteriorly into the interhemi- 
Cortical areas $>40 \mathrm{~mm}^{2}$ with significant differences between patients with Kallmann syndrome and controls by sulcation, curvature, and cortical thickness analyses

\begin{tabular}{|c|c|c|c|c|c|c|c|}
\hline \multirow[b]{2}{*}{ Hemisphere } & \multirow[b]{2}{*}{ Size $\left(\mathrm{mm}^{2}\right)$} & & \multicolumn{3}{|c|}{$\begin{array}{l}\text { Local Maximum, } \\
\text { Talairach }\end{array}$} & \multirow[b]{2}{*}{ FDR Threshold } & \multirow[b]{2}{*}{ Cerebral Region } \\
\hline & & & $x$ & $\mathbf{Y}$ & Z & & \\
\hline \multicolumn{8}{|l|}{ Sulcation } \\
\hline Right & 394.05 & $\downarrow$ & 11.2 & 13.8 & -14.9 & 3.3429 & $\begin{array}{l}\text { Olfactory sulcus and contiguous cortex } \\
\text { of the rectus gyrus }\end{array}$ \\
\hline Right & 325.72 & $\uparrow$ & 16.4 & 28.7 & -23.7 & 3.3429 & Medial orbital-frontal gyrus \\
\hline Left & 518.07 & $\downarrow$ & -9.8 & 16.0 & -15.4 & 3.3159 & $\begin{array}{l}\text { Olfactory sulcus and contiguous cortex of the } \\
\text { rectus gyrus }\end{array}$ \\
\hline Left & 252.41 & $\uparrow$ & -19.6 & 33.2 & -17.3 & 3.3159 & Medial orbital-frontal gyrus \\
\hline \multicolumn{8}{|l|}{ Curvature } \\
\hline Right & 372.85 & $\uparrow$ & 14.4 & 32.3 & -25.3 & 3.4704 & Medial orbital-frontal gyrus \\
\hline Right & 77.61 & $\downarrow$ & 11.7 & 16.6 & -14.2 & 3.4704 & Olfactory sulcus \\
\hline Right & 44.71 & $\downarrow$ & 11.5 & 37.9 & -19.1 & 3.4704 & Olfactory sulcus \\
\hline Left & 196.94 & $\downarrow$ & -11.6 & 38.7 & -20.0 & 3.4704 & Olfactory sulcus \\
\hline Left & 114.79 & $\uparrow$ & -19.6 & 32.7 & -14.9 & 3.4704 & Medial orbital-frontal gyrus \\
\hline \multicolumn{8}{|l|}{ Thickness } \\
\hline Right & 568.97 & $\uparrow$ & -14.1 & 30.7 & -19.2 & 3.3834 & $\begin{array}{l}\text { Olfactory sulcus and contiguous cortex } \\
\text { of the rectus and medial orbital-frontal gyri }\end{array}$ \\
\hline Left & 686.38 & $\uparrow$ & 13.7 & 33.4 & -23.3 & 3.2803 & $\begin{array}{l}\text { Olfactory sulcus and contiguous cortex } \\
\text { of the rectus and medial orbital-frontal gyri }\end{array}$ \\
\hline Left & 78.39 & $\downarrow$ & 12.7 & 48.6 & 33.5 & 3.2803 & Medial orbital-frontal sulcus \\
\hline Left & 40.54 & $\downarrow$ & 45.2 & -62.3 & 36.9 & 3.2803 & Lateral orbital-frontal gyrus \\
\hline
\end{tabular}

Note: $-\uparrow$ indicates that patients with KS showed increased sulcation, curvature, and cortical thickness; $\downarrow$, patients with KS showed decreased sulcation, curvature, and cortical thickness; FDR, false discovery rate.

spheric fissure. ${ }^{7}$ Indeed, as shown in Fig 2, the olfactory sulcus presented with decreased sulcation and curvature, while the medial orbital-frontal sulcus showed a "compensatory-like" increased sulcation and curvature. The concomitant changes of the contiguous orbital-frontal regions seem to highlight a more extensive effect of the olfactory bulbs on forebrain morphogenesis. Most interesting, patients with KS display increased cortical thickness very close to VBM, sulcation, and curvature forebrain anomalies, suggesting that olfactory bulb-induction failure might act not only on the sulcal but also on the structural organization of the cortex.

Regional gray matter increase has been associated with the absence of a sulcus, ${ }^{32}$ though so far no study investigated the correlation between cortical thickness and sulcation abnormalities, to our knowledge. We might speculate that a less deep sulcus results in an abnormal regional gray matter volume and cortical thickness as a consequence of overcrowding neurons migrated to this region. Alternatively, gray matter volume and cortical thickness changes might result from cortical functional differences between patients with KS and controls. The orbital-frontal cortex is deeply involved not only in olfaction (odor identification and olfactory memorization) but also in integrating emotion into cognition within decision-making processes. ${ }^{33} \mathrm{KS}$ is clinically characterized by the absence or reduction of olfaction, whereas few data are available on cognitive functioning and psychiatric risk, though schizophrenic disorders have been anecdotally reported among patients with KS. ${ }^{22,23}$ Nonetheless, the primary and secondary olfactory cortices correspond to piriform and periamygdalar cortices and the posterior orbital-frontal gyrus and insula, all areas that in our study did not present with gray matter and cortical thickness changes. Moreover, none of our patients presented with a history of overt psychiatric disease, even though a specific neuropsychological and psychiatric assessment was not performed. Focused neurocognitive studies are warranted to investigate the presence of basal forebrain function impairment due to the above-mentioned morphologic and structural brain changes.

Finally, the changes observed in our study might be at least partly ascribed to hormonal differences between patients with KS and controls. Most of KS phenotypic features (hypogonadism, small penis, sexual secondary characteristics) are due to low testosterone levels during infancy and adolescence, which could also induce a feminine brain development. Sex brain dimorphism includes brain size, white and gray matter volume and regional cortical thickness changes. ${ }^{34-36}$ This study did not reveal significant differences in brain size between patients with KS and controls. Moreover, sex- and testosterone-related brain differences do not seem to involve the medial orbital-frontal basal regions, thus restraining the direct role of hormonal dysfunction in determining the morphologic pattern observed in patients with KS. Longitudinal studies on patients with KS enrolled before hormone replacement therapy will help clarify the effects of testosterone in postnatal brain development, even though the effect of hormone milieu on prenatal and early brain development phases will remain difficult to unravel.

Because KS is a disease due to mutation of genes that are involved in neuronal migration, ${ }^{37}$ patients with KS have been previously reported to present with midline head and brain abnormalities, such as cleft palate, corpus callosum dysgenesis, and holoprosencephaly. ${ }^{6,38,39}$ These abnormalities have been thoroughly mentioned in studies on patients with KS. ${ }^{7,10,40}$ Nonetheless, brain MR imaging evaluation of our large sample revealed corpus callosum partial agenesis in only $1 / 45$ patients with KS (2.2\%), thus supporting the observation by Quinton et $\mathrm{al}^{7}$ that patients with KS mostly do not present significant intracranial midline abnormalities. A biased inclusion of syndromic patients 
in the initial case series ${ }^{6}$ might explain the difference with more recent larger samples. On the other hand, holoprosencephalic patients might be underdiagnosed as also being affected by hypogonadotropic hypogonadism and hypoanosmia due to their shorter life expectancy and overwhelming clinical issues.

Among our patients with KS, 15 (33\%) showed small nonspecific punctuate FLAIR hyperintensities; 1 showed diffuse multiple sclerosis-like white matter lesions; and 1, an acoustic nerve schwannoma. These findings should be considered incidental unless otherwise demonstrated.

Even though these multimodality MR imaging analyses were applied to a large homogeneous sample of patients with KS with a strictly uniform MR imaging protocol (same scanner, same sequence parameters, and so forth), this study presents some limits. We evaluated KS-related brain MR imaging changes only in males, and further studies are warranted to confirm our findings in female patients with KS. Moreover, the genetic heterogeneity of our sample and its still ongoing molecular characterization did not allow investigating a precise genotypic/MR imaging correlation. Finally, because this was a cross-sectional study with almost no pediatric patients with KS, we could not investigate the brain changes related to aging or the effects of sex hormone deprivation and subsequent replacement on brain development.

The enrollment of specific subgroups, subdivided according to genetics, sex, age, ancillary clinical features (eg, the presence of mirror movement and psychiatric disorders), and treatment will help define more precisely the specific brain changes in KS.

\section{CONCLUSIONS}

This MR imaging study on a large group of male patients with KS showed significant brain changes specifically involving the gyri recti and the contiguous medial orbital-frontal regions. Even though further validation is warranted, curvature, sulcation, cortical thickness, gray/white matter volume, and bone changes in patients with KS point toward a profound structural and morphologic involvement of the basal forebrain that is far more consistent than a simple hypoplasia of the olfactory sulcus, driven by the olfactory bulbs. Future studies on the cognitive and psychiatric domains will define the clinical impact of these morphologic and structural brain changes.

\section{APPENDIX}

Members of the Kallmann Syndrome Neurological Study Group were the following: Arianna D’Errico, “Federico II” University, Napoli, Italy; Giancarlo Ottaviano, University of Padova, Padova, Italy; Elena Cantone, “Federico II” University, Napoli, Italy; Chiara Briani, University of Padova, Padova, Italy; Nella A. Greggio, Azienda Ospeddaliera, University of Padova, Padova, Italy; Silvia Rizzati, University of Padova, Padova, Italy; Marco Rossato, University of Padova, Padova, Italy; Eugenio De Carlo, University of Padova, Padova, Italy; Elvira Napoli, Medicanova Diagnostic Center, Battipaglia, Italy; Gianfranco D’Agosto, Medicanova Diagnostic Center, Battipaglia, Italy; Giangennaro Coppola, University of Salerno, Salerno, Italy.

Disclosures: Renzo Manara_UNRELATED: Payment for Lectures (including service on Speakers Bureaus): BioMarin, Comments: lecture on mucopolysaccharidosis type 6 (600 Euro).

\section{REFERENCES}

1. Seminara SB, Hayes FJ, Crowley WF Jr. Gonadotropin-releasing hormone deficiency in the human (idiopathic hypogonadotropic hypogonadism and Kallmann's syndrome): pathophysiological and genetic considerations. Endocr Rev 1998;19:521-39

2. Kallmann FJ, Schonfeld WA, Barvera SE. The genetic aspects of primary eunuchoidism. Am J Ment Defic 1944;48:203-36

3. Schwanzel-Fukuda M, Bick D, Pfaff DW. Luteinizing hormone-releasing hormone (LHRH)-expressing cells do not migrate normally in an inherited hypogonadal (Kallmann) syndrome. Brain Res $\mathrm{Mol}$ Brain Res 1989;6:311-26

4. Okubo K, Sakai F, Lau EL, et al. Forebrain gonadotropin-releasing hormone neuronal development: insights from transgenic medaka and the relevance to $\mathrm{X}$-linked Kallmann syndrome. Endocrinology 2006;147:1076-84

5. Maestre de San Juan A. Falta total de los nervios olfactorios con anosmía en un individuo en quien existia una atrofía congénita de los testículos y miembro viril. Siglo Medico 1856;131-211

6. De Morsier G. Studies in cranio-encephalic dysraphia. I. Agenesia of the olfactory lobe (lateral telencephaloschisis) and of the callous and anterior commissures (median telencephaloschisis); olfactogenital dysplasia [in French]. Schweiz Arch Neurol Psychiatr 1954;74:309-61

7. Quinton R, Duke VM, de Zoysa PA, et al. The neuroradiology of Kallmann's syndrome: a genotypic and phenotypic analysis. J Clin Endocrinol Metab 1996;81:3010-17

8. Birnbacher R, Wandl-Vergesslich K, Frisch H. Diagnosis of X-recessive Kallmann syndrome in early infancy: evidence of hypoplastic rhinencephalon. Eur J Pediatr 1994;153:245-47

9. Truwit CL, Barkovich AJ, Grumbach MM, et al. MR imaging of Kallmann syndrome, a genetic disorder of neuronal migration affecting the olfactory and genital systems. AJNR Am J Neuroradiol 1993;14:827-38

10. Vogl TJ, Stemmler J, Heye B, et al. Kallman syndrome versus idiopathic hypogonadotropic hypogonadism at MR imaging. Radiology 1994;191:53-57

11. Yousem DM, Turner WJ, Li C, et al. Kallmann syndrome: MR evaluation of olfactory system. AJNR Am J Neuroradiol 1993;14:839-43

12. Knorr JR, Ragland RL, Brown RS, et al. Kallmann syndrome: MR findings. AJNR Am J Neuroradiol 1993;14:845-51

13. Koenigkam-Santos M, Santos AC, Versiani BR, et al. Quantitative magnetic resonance imaging evaluation of the olfactory system in Kallmann syndrome: correlation with a clinical smell test. Neuroendocrinology 2011;94:209-17

14. de m Freitas P, Carvalho S, Ribeiro F, et al. Neuroradiology of Kallmann's syndrome [in Portuguese]. Acta Med Port 2001;14:123-26

15. Maione L, Benadjaoud S, Eloit C, et al. Computed tomography of the anterior skull base in Kallmann syndrome reveals specific ethmoid bone abnormalities associated with olfactory bulb defects. J Clin Endocrinol Metab 2013;98:E537-46

16. Ueno H, Yamaguchi H, Katakami H, et al. A case of Kallmann syndrome associated with Dandy-Walker malformation. Exp Clin Endocrinol Diabetes 2004;112:62-67

17. Koenigkam-Santos M, Santos AC, Borduqui T, et al. Whole-brain voxel-based morphometry in Kallmann syndrome associated with mirror movements. AJNR Am J Neuroradiol 2008;29:1799-804

18. Costa-Barbosa FA, Balasubramanian R, Keefe KW, et al. Prioritizing genetic testing in patients with Kallmann syndrome using clinical phenotypes. J Clin Endocrinol Metab 2013;98:E943-53

19. Dale AM, Fischl B, Sereno MI. Cortical surface-based analysis. I. Segmentation and surface reconstruction. Neuroimage 1999;9:179-94

20. Fischl B, Sereno MI, Dale AM. Cortical surface-based analysis. II: inflation, flattening, and a surface-based coordinate system. $\mathrm{Neu}$ roimage 1999;9:195-207

21. Fischl B, Dale AM. Measuring the thickness of the human cerebral cortex from magnetic resonance images. Proc Natl Acad Sci U S A 2000;97:11050-55 
22. Vagenakis GA, Hyphantis TN, Papageorgiou C, et al. Kallmann's syndrome and schizophrenia. Int J Psychiatry Med 2004;34:379-90

23. Verhoeven WM, Egger JI, Hovens JE, et al. Kallmann syndrome and paranoid schizophrenia: a rare combination. BMJ Case Rep 2013; 2013: pii: bcr2012007387

24. Oldfield RC. The assessment and analysis of handedness: the Edinburgh inventory. Neuropsychologia 1971;9:97-113

25. Ashburner J, Friston KJ. Voxel-based morphometry: the methods. Neuroimage 2000;11(6 pt 1):805-21

26. Maldjian JA, Laurienti PJ, Kraft RA, et al. An automated method for neuroanatomic and cytoarchitectonic atlas-based interrogation of FMRI data sets. Neuroimage 2003;19:1233-39

27. Smith SM, Nichols TE. Threshold-free cluster enhancement: addressing problems of smoothing, threshold dependence and localisation in cluster inference. Neuroimage 2009;44:83-98

28. Krams M, Quinton R, Ashburner J, et al. Kallmann's syndrome: mirror movements associated with bilateral corticospinal tract hypertrophy. Neurology 1999;52:816-22

29. Schwob JE, Szumowski KE, Leopold DA, et al. Histopathology of olfactory mucosa in Kallmann's syndrome. Ann Otol Rhinol Laryngol 1993;102:117-22

30. Abolmaali ND, Hietschold V, Vogl TJ, et al. MR evaluation in patients with isolated anosmia since birth or early childhood. AJNR Am J Neuroradiol 2002;23:157-64

31. Nguyen AD, Pelavin PE, Shenton ME, et al. Olfactory sulcal depth and olfactory bulb volume in patients with schizophrenia: an MRI study. Brain Imaging Behav 2011;5:252-61
32. Buda M, Fornito A, Bergström ZM, et al. A specific brain structural basis for individual differences in reality monitoring. $J$ Neurosci 2011;31:14308-13

33. Bechara A, Damasio H, Damasio AR. Emotion, decision making and the orbitofrontal cortex. Cereb Cortex 2000;10:295-307

34. Sacher J, Neumann J, Okon-Singer H, et al. Sexual dimorphism in the human brain: evidence from neuroimaging. Magn Reson Imaging 2013;31:366-75

35. Witte AV, Savli M, Holik A, et al. Regional sex differences in grey matter volume are associated with sex hormones in the young adult human brain. Neuroimage 2010;49:1205-12

36. Im K, Lee JM, Lee J, et al. Gender difference analysis of cortical thickness in healthy young adults with surface-based methods. Neuroimage 2006;31:31-38

37. Bonomi M, Libri DV, Guizzardi F, et al, for the Idiopathic Central Hypogonadism Study Group of the Italian Societies of Endocrinology and Pediatric Endocrinology and Diabetes. New understandings of the genetic basis of isolated idiopathic central hypogonadism. Asian J Androl 2012;14:49-56

38. Parr JH. Midline cerebral defects and Kallmann's syndrome. JR Soc Med 1988;81:355-56

39. Gibby OM, Rayner PHW, West RJ, et al. Short stature due to median craniocephalic dysraphia with nasal encephalocoele. In: Proceedings of the 1st Meeting of the British Endocrine Society, 1982; Abstract 98:48

40. Dodé C, Hardelin JP. Kallmann syndrome. Eur J Hum Genet 2009; $17: 139-46$ 